\title{
Generalised BGP reflection functors via the Grothendieck construction
}

\author{
Tobias Dyckerhoff, Gustavo Jasso and Tashi Walde
}

\begin{abstract}
Inspired by work of Ladkani, we explain how to construct generalisations of the classical reflection functors of Bernštel̆n, Gel'fand and Ponomarev by means of the Grothendieck construction.
\end{abstract}

\section{Introduction}

Reflection functors were introduced to representation theory by Bernštel̆n, Gel'fand, and Ponomarev in the seminal article [BGP73] to provide a more transparent proof of Gabriel's Theorem [Gab72]. After fundamental work of Brenner and Butler [BB76], the BGP reflection functors were extended by Auslander, Platzeck and Reiten APR79] to Artin algebras with a simple projective (resp. injective) module. It was then observed by Happel Hap87 that BGP reflection functors can be understood conceptually in terms of derived equivalences induced by what nowadays are called APR tilting complexes. These equivalences have been used, for example, to show that the derived category of representations of a finite quiver whose underlying graph is a tree does not depend on its orientation.

Generalising the BGP reflection functors for tree quivers, Ladkani Lad07a constructed equivalences between derived categories of representations of finite posets in an arbitrary abelian category. Ladkani uses these 'generalised BGP reflection functors' to establish derived equivalences between posets arising naturally in representation theory and combinatorics Lad07b, Lad07c, Lad08a. In a similar spirit, abstract versions of the BGP reflection functors were developed by Groth and Štovíček in a series of articles GŠ18b, GŚ16a, GŠ16b with the most general form of their construction appearing as the main result in GS18a.

In this article we construct generalised BGP reflection functors by leveraging a general equivalence between two stable $\infty$-categories associated to an exact functor via gluing operations, thereby unifying the aforementioned approaches. From the perspective of semi-orthogonal decompositions [BK89, these generalised BGP reflection functors can be interpreted as a mutation between the two decompositions associated to an admissible subcategory of a stable $\infty$-category. Note that the mentioned gluing operations cannot be performed with triangulated categories and thus we are required to pass to a richer framework such as that of Lurie's stable $\infty$-categories.

In Section 1 we recall two - equivalent-procedures to glue stable $\infty$-categories along an exact functor. In Section 2 we construct the generalised BGP reflection functors (Theorem 2.3) and discuss applications and examples.

Acknowledgements. The authors thank Catharina Stroppel for detailed and valuable comments on an earlier version of this article. T.D. acknowledges the support by the VolkswagenStiftung for his Lichtenberg Professorship at the University of Hamburg; T.W. was supported by a Hausdorff Scholarship from the Bonn International Graduate School (BIGS) of Mathematics.

\section{Preliminaries: gluing along an exact functor}

Recall that a pointed $\infty$-category $\mathcal{C}$ with finite limits and finite colimits is stable if the suspension functor $\Sigma_{\mathfrak{C}}: \mathcal{C} \rightarrow \mathcal{C}$ and the loop functor $\Omega_{\mathfrak{C}}: \mathcal{C} \rightarrow \mathcal{C}$, given by

$$
\Sigma_{\mathcal{e}}: c \mapsto \operatorname{cofib}(c \rightarrow 0) \quad \text { and } \quad \Omega_{\mathcal{e}}: c \mapsto \operatorname{fib}(0 \rightarrow c),
$$

respectively, are mutually quasi-inverse equivalences, see Proposition 1.4.2.11 in Lur17. The following construction is the raison d'être for the use of stable $\infty$-categories in this article. 
Construction 1.1. Let $F: \mathcal{B} \rightarrow \mathcal{A}$ be an exact functor between stable $\infty$-categories.

1. Let $\mathcal{L}_{\star}(F)$ be the $\infty$-category defined by the following pullback diagram in the (very large) $\infty$-category $\mathrm{CAT}_{\infty}$ of $\infty$-categories:

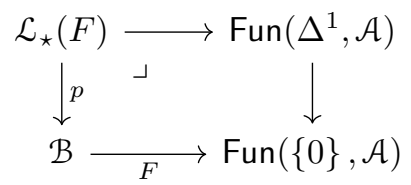

Thus, informally, an object of $\mathcal{L}_{\star}(F)$ can be identified with a triple

$$
(b, F(b) \rightarrow a)
$$

where $b$ is an object of $\mathcal{B}, a$ is object of $\mathcal{A}$, and $F(b) \rightarrow a$ is a morphism in $\mathcal{A}$. The $\infty$-category $\mathcal{L}_{\star}(F)$ is stable as it is a limit of stable $\infty$-categories and exact functor ${ }^{1}$.

2. Dually, we define $\mathcal{L}^{\star}(F)$ to be the (stable) $\infty$-category defined by the following pullback diagram in $\mathrm{CAT}_{\infty}$ :

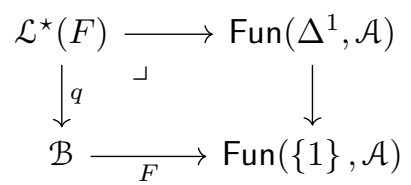

Thus, informally, an object of $\mathcal{L}^{\star}(F)$ can be identified with a triple

$$
(b, a \rightarrow F(b))
$$

where $b$ is an object of $\mathcal{B}, a$ is an object of $\mathcal{A}$, and $a \rightarrow F(b)$ is a morphism in $\mathcal{A}$.

Remark 1.2. The stable $\infty$-category $\mathcal{L}_{\star}(F)$ (resp. $\mathcal{L}^{\star}(F)$ ) associated to an exact functor $F$ can be identified ${ }^{2}$ with the stable $\infty$-category of sections of the contravariant (resp. covariant) Grothendieck construction. See Section 3.2 in Lur09 for more details on the Grothendieck construction.

Lemma 1.3. Let $F: \mathcal{B} \rightarrow \mathcal{A}$ be an exact functor between stable $\infty$-categories. There are mutually quasi-inverse equivalences

$$
S^{-}: \mathcal{L}^{\star}(F) \rightleftarrows \mathcal{L}_{\star}(F): S^{+}
$$

which, informally, are given by

$$
\begin{aligned}
& S^{-}:(b, \varphi: a \rightarrow F(b)) \longmapsto(b, F(b) \rightarrow \operatorname{cofib}(\varphi)), \\
& S^{+}:(b, \psi: F(b) \rightarrow a) \longmapsto(b, \operatorname{fib}(\psi) \rightarrow F(b)) .
\end{aligned}
$$

Proof. Consider the solid commutative diagram of stable $\infty$-categories and exact functors

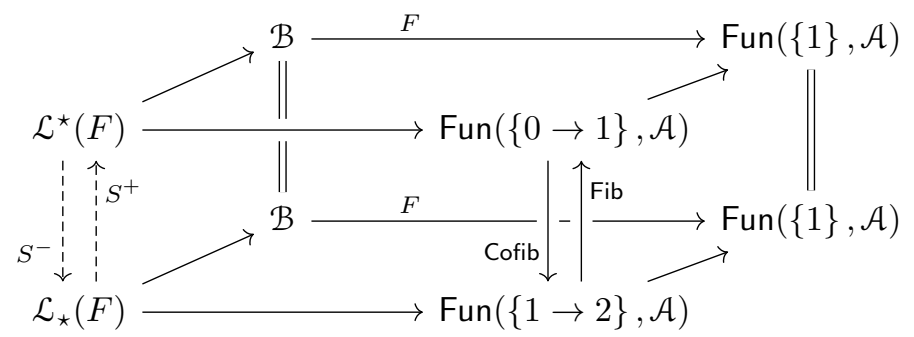

where the bottom and top squares are the pullback squares (1) and (2), respectively. Since Cofib and Fib are mutually quasi-inverse equivalences, the functoriality of pullbacks implies the existence of the desired mutually quasi-inverse equivalences $S^{-}$and $S^{+}$which render the above cube commutative.

\footnotetext{
${ }^{1}$ The $\infty$-category of stable $\infty$-categories is closed under limits of $\infty$-categories, see Theorem 1.1.4.4 in Lur17.

${ }^{2}$ See for instance Lemma 5.4.7.15 in Lur09].
} 
The statement of Lemma 1.3 has the following interpretations in terms of the classical concepts of recollements and semi-orthogonal decompositions.

Remark 1.4. Recollements were introduced by Beĭlinson, Bernšter̆n and Deligne in BBD82 in the language of triangulated categories. It has since been observed that, when working with enhanced triangulated categories, recollements can be recovered from their gluing functors. For example, using differential graded categories as enhancements - as proposed in [BK90] - the relevant theory is developed systematically in [KL15]; a treatment in the language of stable $\infty$-categories can be found in Appendix A.8 in Lur17. Recall that a recollement is a diagram of stable $\infty$-categories and exact functors of the form

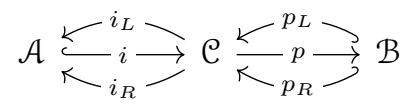

such that the following conditions are satisfied:

1. The functor $i$ is fully faithful and its essential image is precisely the kernel of $p$. Moreover, there are adjunctions $i_{L} \dashv i \dashv i_{R}$.

2. There are adjunctions $p_{L} \dashv p \dashv p_{R}$ and the functors $p_{L}$ and $p_{R}$ are fully faithful.

The gluing functor of a recollement of the form $(3)$ is the exact functor $i_{R} \circ p_{L}: \mathcal{B} \rightarrow \mathcal{A}$. The results of Appendix A.8 in Lur17] imply that the forgetful functor which associates to a recollement of stable $\infty$-categories its gluing functor induces an equivalence of $\infty$-categories between the $\infty$-category of recollements and the $\infty$-category $\operatorname{Fun}\left(\Delta^{1}\right.$, Cat $\left._{\infty}^{\mathrm{ex}}\right)$ of exact functors between stable $\infty$-categories. Construction 1.1 provides two possible quasi-inverses to this equivalence while Lemma 1.3 provides a canonical identification between these quasi-inverses. Indeed, for an exact functor $F: \mathcal{B} \rightarrow \mathcal{A}$ between stable $\infty$-categories, the functors $S^{-}$and $S^{+}$induce mutually quasi-inverse equivalences of recollements

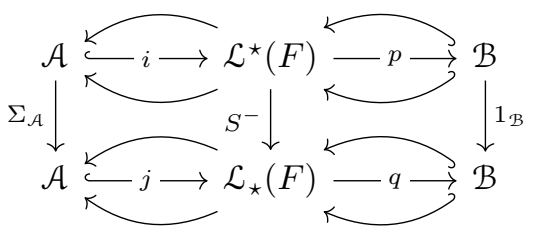

and

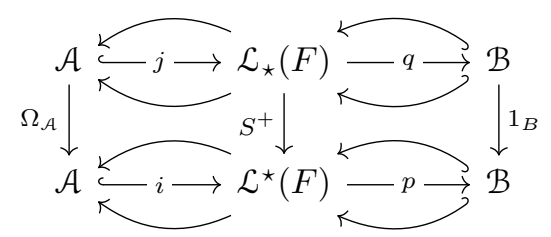

Remark 1.5. Semi-orthogonal decompositions were introduced by Bondal and Kapranov in [BK89] in the language of triangulated categories. In their language, the datum of a recollement with middle term $\mathcal{C}$ is equivalent ${ }^{3}$ to the datum of the inclusion $\mathcal{A} \subset \mathcal{C}$ of an admissible subcategory, that is a subcategory such that the inclusion has left and right adjoints. To such a subcategory correspond two semi-orthogonal decompositions $\left\langle\mathcal{A}^{\perp}, \mathcal{A}\right\rangle$ and $\left\langle\mathcal{A},{ }^{\perp} \mathcal{A}\right\rangle$ of $\mathcal{C}$ which are mutations of one another. In terms of the recollement data, the corresponding orthogonals are given by $\mathcal{A}^{\perp}=p_{R}(\mathcal{B})$ and ${ }^{\perp} \mathcal{A}=p_{L}(\mathcal{B})$. Let $F:=i_{R} \circ p_{L}: \mathcal{B} \rightarrow \mathcal{A}$ be the gluing functor.

1. The stable $\infty$-category $\mathcal{L}_{\star}(F)$ is canonically equivalent to the $\infty$-category of arrows in $\mathcal{C}$ from $\mathcal{A}^{\perp}$ to $\mathcal{A}$ and hence, via the fibre functor, also equivalent to $\mathcal{C}$ itself.

2. The stable $\infty$-category $\mathcal{L}^{\star}(F)$ is canonically equivalent to the $\infty$-category of arrows in $\mathcal{C}$ from $\mathcal{A}$ to ${ }^{\perp} \mathcal{A}$ and hence, via the cofibre functor, also equivalent to $\mathcal{C}$ itself.

Hence, the resulting equivalence $\mathcal{L}_{\star}(F) \simeq \mathcal{L}^{\star}(F)$ (which agrees with the one from Lemma 1.3) can be interpreted as passing from the description of the category $\mathcal{C}$ in terms of the semi-orthogonal decomposition $\left\langle\mathcal{A}^{\perp}, \mathcal{A}\right\rangle$ to a description in terms of the mutated decomposition $\left\langle\mathcal{A},{ }^{\perp} \mathcal{A}\right\rangle$. In particular, from this perspective, the generalised BGP reflection functors constructed below arise from mutations of semi-orthogonal decompositions.

\footnotetext{
${ }^{3}$ See for instance Proposition A.8.20 in [Lur17].
} 


\section{Generalised BGP reflection functors}

We fix a stable $\infty$-category $\mathcal{D}$ throughout this section. For a small $\infty$-category $Z$ we denote by $\mathcal{D}(Z)$ the $\infty$-category $\operatorname{Fun}(Z, \mathcal{D})$ of $Z$-shaped diagrams in $\mathcal{D}$. Our aim is to construct equivalences of the form

$$
S^{-}: \mathcal{D}(Z) \rightleftarrows \mathcal{D}(S): S^{+}
$$

using the functors of Lemma 1.3, where $S$ is obtained from $Z$ by 'reflecting some arrows'. When $Z$ is a quiver or a poset and $\mathcal{D}$ is the derived $\infty$-category of vector spaces over a field, these equivalences reduce - after passing to homotopy categories - to triangle equivalences between derived categories of representations. This is a consequence of the following general fact, see for example Proposition 4.2.4.4 in Lur09.

Fact. Let $\mathcal{D}_{\mathcal{A}}$ be the derived $\infty$-category of a Grothendieck category $\mathcal{A}$. The derived category of the Grothendieck category $\mathcal{A}^{Z}$ of $Z$-shaped diagrams in $\mathcal{A}$ is equivalent to the homotopy category of the stable $\infty$-category $\mathcal{D}_{\mathcal{A}}(Z)$.

\subsection{The main theorem}

Let $Q=\left(Q_{0}, Q_{1}\right)$ be a quiver. For a $Q$-shaped diagram $\mathcal{F}: Q \rightarrow$ Cat $_{\infty}$ of small $\infty$-categories, we denote the covariant (resp. contravariant) Grothendieck construction of $\mathcal{F}$ by $\int_{Q} \mathcal{F}$ (resp. $\int^{Q} \mathcal{F}$ ); we refer the reader to Section 3.2.5 in [Lur09] for detail.4. Following Definition 4.12 in [Lad08b], we say that $Q$ is bipartite if there exists a decomposition $Q_{0}=V \dot{\cup} W$ such that all arrows are of the form $v \rightarrow w$ for $v \in V$ and $w \in W$. If $Q$ is bipartite, then the (small) $\infty$-categories $\int_{Q} \mathcal{F}$ and $\int^{Q} \mathcal{F}$ are

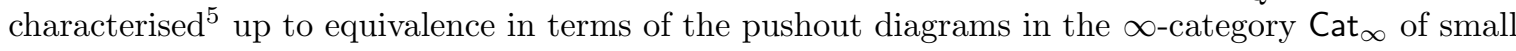
$\infty$-categories

$$
\begin{aligned}
& \coprod_{\alpha: v \rightarrow w} \mathcal{F} v \longrightarrow \coprod_{w \in W} \mathcal{F} w \\
& \downarrow \quad\ulcorner\downarrow \downarrow \text { and } \\
& \coprod_{v \in V} \mathcal{F} v \times Q_{v /} \longrightarrow \int_{Q} \mathcal{F} \\
& \coprod_{\alpha: v \rightarrow w} \mathcal{F} v \longrightarrow \coprod_{w \in W} \mathcal{F} w \\
& \downarrow \quad\ulcorner\quad \downarrow \\
& \coprod_{v \in V} \mathcal{F} v \times Q^{\mathrm{op}} / v \rightarrow \int^{Q} \mathcal{F}
\end{aligned}
$$

where $Q_{v /}$ (resp. $Q^{\mathrm{op}} / v$ ) denotes the slice category of objects under (resp. over) $v$. In the case $Q=\{0 \rightarrow 1\}$, corresponding to a functor $f: X \rightarrow Y$ between small $\infty$-categories, the $\infty$-categories $\int_{Q} \mathcal{F}$ and $\int^{Q} \mathcal{F}$ can be schematically illustrated as follows:
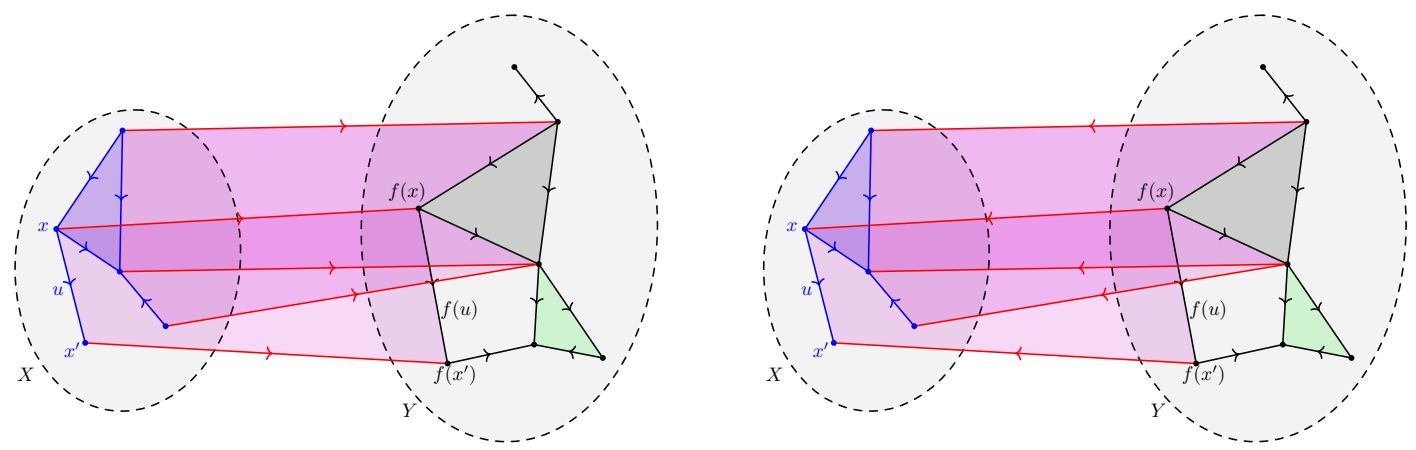

The coloured regions indicate commutativity relations.

Remark 2.1. If the diagram $\mathcal{F}$ takes values in ordinary categories, then $\int_{Q} \mathcal{F}$ and $\int^{Q} \mathcal{F}$ agree with (the nerve of) the corresponding 1-categorical Grothendieck constructions. If $\mathcal{F}$ furthermore takes values in posets, then $\int_{Q} \mathcal{F}$ (resp. $\int^{Q} \mathcal{F}$ ) is again a poset if and only if, for every pair $\alpha, \beta: v \rightarrow w$ of parallel arrows in $Q$ and every $x \in \mathcal{F} v$, the elements $(\mathcal{F} \alpha)(x)$ and $(\mathcal{F} \beta)(x)$ of $\mathcal{F} w$ have no common

\footnotetext{
${ }^{4}$ What we refer to as the Grothendieck construction is called the relative nerve in Lur09]; the explicit construction is given in Definition 3.2.5.2.

${ }^{5}$ Using the fact that $Q$ is bipartite, these are direct applications of Theorem 7.4 and Corollary 7.6 in GHN17] (after unravelling the notation).
} 
upper (resp. lower) bound. Under these assumptions - which are precisely the assumptions in the main theorem in Lad07a - the Grothendieck construction specialises to the construction therein.

Construction 2.2. Let $Q$ be a finite bipartite quiver with $Q_{0}=V \dot{\cup} W$ and $\mathcal{F}: Q \rightarrow$ Cat $_{\infty}$ a $Q$-shaped diagram of small $\infty$-categories. We define $\infty$-categories

$$
X:=\coprod_{v \in V} \mathcal{F} v \quad \text { and } \quad Y:=\coprod_{w \in W} \mathcal{F} w
$$

and an exact functor $F=F_{\mathcal{F}}: \mathcal{D}(Y) \rightarrow \mathcal{D}(X)$ as the composite

$$
\mathcal{D}(Y) \stackrel{\simeq}{\longrightarrow} \bigoplus_{w \in W} \mathcal{D}(\mathcal{F} w) \stackrel{(-\circ \mathcal{F} \alpha)_{\alpha}}{\longrightarrow} \bigoplus_{\alpha: v \rightarrow w} \mathcal{D}(\mathcal{F} v) \stackrel{\oplus}{\longrightarrow} \bigoplus_{v \in V} \mathcal{D}(\mathcal{F} v) \stackrel{\simeq}{\longrightarrow} \mathcal{D}(X) .
$$

In particular, for $M \in \bigoplus_{w \in W} \mathcal{D}(\mathcal{F} w)$ and $x \in \mathcal{F} v$ we have

$$
(F M)_{x}=\bigoplus_{\alpha: v \rightarrow w} M_{(\mathcal{F} \alpha)(x)}
$$

The following result extends Ladkani's main theorem in [Lad07a] from posets to small $\infty$-categories.

Theorem 2.3. In the setting of Construction 2.2, there are canonical equivalences of stable $\infty$ categories

$$
\mathcal{L}^{\star}(F) \simeq \mathcal{D}\left(\int_{Q} \mathcal{F}\right) \quad \text { and } \quad \mathcal{L}_{\star}(F) \simeq \mathcal{D}\left(\int^{Q} \mathcal{F}\right)
$$

In particular, the functors of Lemma 1.3 induce mutually quasi-inverse equivalences of stable $\infty$ categories

$$
S^{-}: \mathcal{D}\left(\int_{Q} \mathcal{F}\right) \rightleftarrows \mathcal{D}\left(\int^{Q} \mathcal{F}\right): S^{+}
$$

We call these equivalences generalised BGP reflection functors.

Proof. We only establish the existence of the leftmost equivalence in (5), the other one being analogous. Note that the existence of the desired equivalences (6) then follows immediately from Lemma 1.3.

Firstly, applying the functor $\mathcal{D}(-)=\operatorname{Fun}(-, \mathcal{D})$ - which take ${ }^{6}$ pushouts in Cat $_{\infty}$ to pullbacks in $\mathrm{CAT}_{\infty}$ - to the diagram (4) we see that the leftmost square in the diagram

$$
\begin{gathered}
\mathcal{D}\left(\int_{Q} \mathcal{F}\right) \longrightarrow \mathcal{D}\left(\coprod_{v \in V} \mathcal{F} v \times Q_{v /}\right) \longrightarrow \mathcal{D}\left(\coprod_{v \in V} \mathcal{F} v \times \Delta^{1}\right) \simeq \operatorname{Fun}\left(\Delta^{1}, \mathcal{D}(X)\right) \\
\quad \downarrow \\
\quad \stackrel{\downarrow}{\downarrow}(Y) \longrightarrow \mathcal{D}\left(\coprod_{\alpha: v \rightarrow w} \mathcal{F} v\right) \longrightarrow \mathcal{D}\left(\coprod_{v \in V} \mathcal{F} v \times\{1\}\right) \simeq \operatorname{Fun}(\{1\}, \mathcal{D}(X))
\end{gathered}
$$

is a pullback of $\infty$-categories, where the bottom horizontal composite is precisely the functor $F$. Secondly the rightmost square can be identified with the pullback square

$$
\begin{gathered}
\bigoplus_{v \in V} \operatorname{Fun}\left(Q_{v /}, \mathcal{D}(\mathcal{F} v)\right) \longrightarrow \\
\downarrow \\
\bigoplus_{v \in V} \bigoplus_{\alpha: v \rightarrow w} \mathcal{D}(\mathcal{F} v) \underset{\ominus}{\longrightarrow} \bigoplus_{v \in V} \operatorname{Fun}\left(\Delta^{1}, \mathcal{D}(\mathcal{F} v)\right) \\
\downarrow
\end{gathered}
$$

Therefore the outer rectangle in (7) is also a pullback square. Finally, the claim follows by comparison with the pullback square $[2]$ defining $\mathcal{L}^{\star}(F)$.

\footnotetext{
${ }^{6}$ Indeed, limits in $\mathrm{CAT}_{\infty}$ can be detected after applying the functors Map $_{\mathrm{CAT}_{\infty}}(z,-):$ CAT $\infty \rightarrow$ SPACES for $z \in$ $\mathrm{CAT}_{\infty}$ and the functor $\operatorname{Map}_{\mathrm{CAT}_{\infty}}(Z, \mathcal{D}(-))$ is equivalent to the representable functor Map $\mathrm{CAT}_{\infty}(-, \mathcal{D}(Z))$ which sends colimits to limits.
} 


\subsection{Applications}

We now explain how to recover the main result in GŠ18a (see Corollary 2.6 below) as well as further results from Lad07a (see Corollary 2.8 and Corollary 2.10 below) as special cases of Theorem 2.3.

We begin by highlighting the most important instance of Theorem 2.3. Let $K_{d}$ be the $d$-Kronecker quiver, that is the category with two objects 0,1 and $d$ parallel arrows $0 \rightarrow 1$. Let $f_{1}, \ldots, f_{d}: X \rightarrow Y$ be functors between small $\infty$-categories and denote by $\int_{K_{d}} f$ and $\int{ }^{K_{d}} f$ the covariant and contravariant Grothendieck constructions of the corresponding $K_{d}$-shaped diagram of $\infty$-categories.

Corollary 2.4. There are mutually quasi-inverse equivalences of stable $\infty$-categories

$$
S^{-}: \mathcal{D}\left(\int_{K_{d}} f\right) \rightleftarrows \mathcal{D}\left(\int^{K_{d}} f\right): S^{+}
$$

induced by the functors of Lemma 1.3 .

Proof. Apply Theorem 2.3 to the (bipartite) quiver $K_{d}$.

Example 2.5. Let $X=\{0 \rightarrow 1\}$ and $Y=\{0 \rightarrow 1 \rightarrow 2\}$ and consider the functors $f_{1}, f_{2}: X \rightarrow$ $Y$ given by $f_{1}(0)=0$ and $f_{1}(1)=1$, and $f_{2}(0)=f_{2}(1)=2$. The covariant and contravariant Grothendieck constructions $\int_{K_{d}} f$ and $\int^{K_{d}} f$ of the resulting diagram $f: K_{d} \rightarrow$ Cat are as follows:
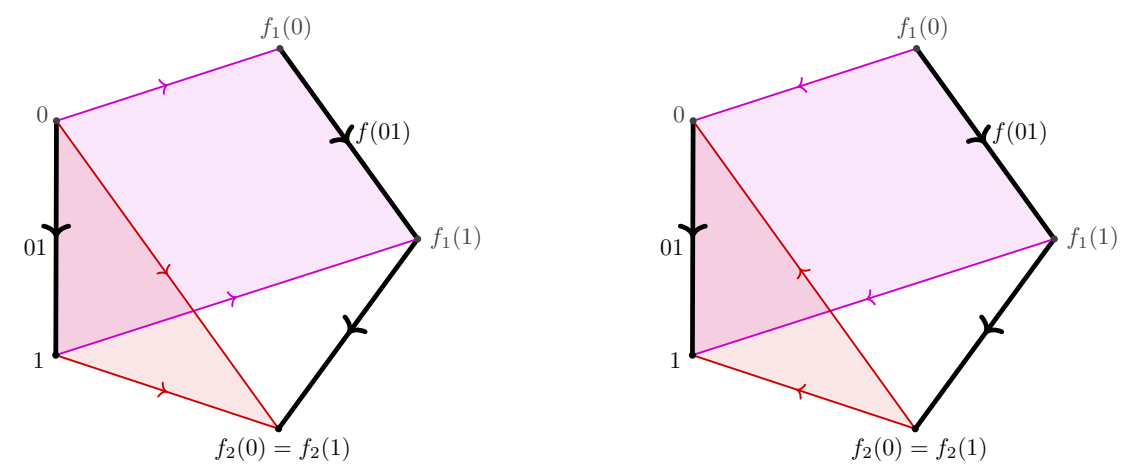

The coloured regions indicate commutativity relations.

The following result recovers the classical BGP reflection functors by letting $\mathcal{D}$ be the derived $\infty$-category of vector spaces over a field.

Corollary 2.6 (Classical BGP reflection functors). Let $Y$ be a small $\infty$-category and choose finitely many objects $y_{1}, \ldots, y_{d} \in Y$ classified by functors $f_{1}, \ldots, f_{d}: * \rightarrow Y$. There are mutually quasiinverse equivalences

$$
S^{-}: \mathcal{D}\left(\int_{K_{d}} f\right) \rightleftarrows \mathcal{D}\left(\int^{K_{d}} f\right): S^{+}
$$

induced by the functors of Lemma 1.3, where $f: K_{d} \rightarrow \mathrm{Cat}_{\infty}$ is the associated $K_{d}$-shaped diagram.

Proof. This is the case of Corollary 2.4 where $X$ is a point.

Remark 2.7. In the setting of Corollary 2.6. the $\infty$-categories $\int_{K_{d}} f$ and $\int^{K_{d}} f$ are obtained from $Y$ by adjoining a new source (resp. sink) $y$ with free arrows $y \rightarrow y_{i}$ (resp. $y_{i} \rightarrow y$ ). In particular, if $Y$ is a poset, then $\int_{K_{d}} f$ and $\int^{K_{d}} f$ are almost never posets. The functor $S^{-}$acts as follows: Given a representation

the representation

$$
M: \int_{K_{d}} f \longrightarrow \mathcal{D}
$$

$$
S^{-} M: \int^{K_{d}} f \longrightarrow \mathcal{D}
$$

is given by $\left(S^{-} M\right)_{y^{\prime}}=M_{y^{\prime}}$ if $y^{\prime} \neq y$ and

$$
\left(S^{-} M\right)_{y}=\operatorname{cofib}\left(M_{y} \rightarrow \bigoplus_{i} M_{y_{i}}\right) .
$$

The action of $S^{+}$can be described similarly, in terms of the fibre functor. This description is in complete analogy with the classical BGP reflection functors; moreover, one can show that it agrees with the abstract reflection functors of GŚ18a. 
The following result extends Corollary 1.3 in Lad07a from posets to small $\infty$-categories.

Corollary 2.8. Let $f: X \rightarrow Y$ be a functor between small $\infty$-categories. There are mutually quasiinverse equivalences

$$
S^{-}: \mathcal{D}\left(\int_{\Delta^{1}} f\right) \rightleftarrows \mathcal{D}\left(\int^{\Delta^{1}} f\right): S^{+}
$$

induced by the functors of Lemma 1.3.

Proof. This is the case $d=1$ of Corollary 2.4 .

Example 2.9. Let $X=\{0 \rightarrow 1 \rightarrow 2\}$ and $Y=\{0 \rightarrow 1\}$ and consider the functor $f: X \rightarrow Y$ given by $f(0)=f(1)=0$ and $f(2)=1$. The covariant and contravariant Grothendieck constructions $\int_{\Delta^{1}} f$ and $\int^{\Delta^{1}} f$ of the induced diagram $f: \Delta^{1} \rightarrow$ Cat are as follows:
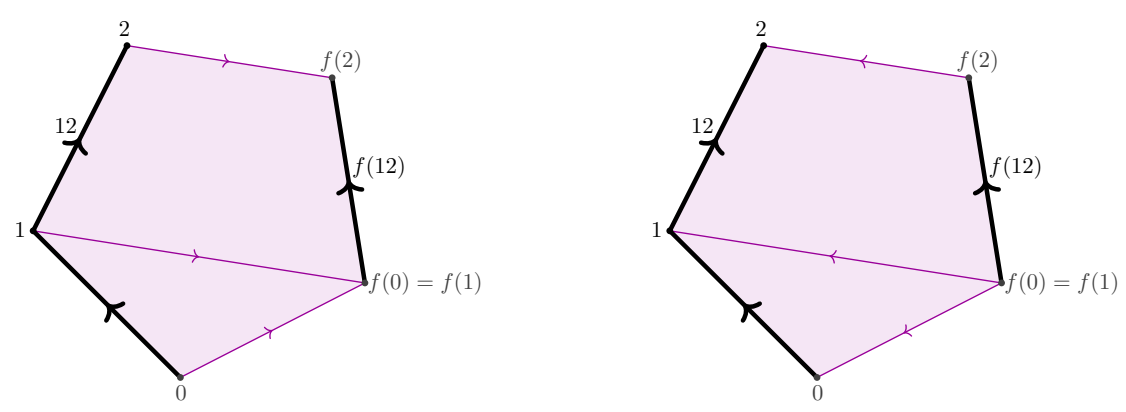

The coloured regions indicate commutativity relations.

The following result extends Corollary 1.5 in Lad07a] from posets to small $\infty$-categories. Recall that, given an $\infty$-category $X$, there are $\infty$-categories $X^{\triangleright}$ and $X^{\triangleleft}$ obtained by adding to $X$ a terminal object $\infty$ or an initial object $-\infty$, respectively.

Corollary 2.10. Let $X$ be a small $\infty$-category. There are mutually quasi-inverse equivalences

$$
S^{-}: \mathcal{D}\left(X^{\triangleright}\right) \rightleftarrows \mathcal{D}\left(X^{\triangleleft}\right): S^{+}
$$

induced by the functors of Lemma 1.3 .

Proof. Apply Corollary 2.8 to the unique functor $f: X \rightarrow *$ and note that $\int_{\Delta^{1}} f$ and $\int^{\Delta^{1}} f$ are equivalent to $X^{\triangleright}$ and $X^{\triangleleft}$, respectively.

Remark 2.11. In the situation of Corollary 2.10 the functor $S^{-}$acts as follows: For a representation

$$
M: X^{\triangleright} \longrightarrow \mathcal{D},
$$

the representation

$$
S^{-} M: X^{\triangleleft} \longrightarrow \mathcal{D}
$$

is given by $\left(S^{-} M\right)_{-\infty}=M_{\infty}$ and

$$
\left(S^{-} M\right)_{x}=\operatorname{cofib}\left(M_{x} \rightarrow M_{\infty}\right)
$$

for each object $x$ of $X$. The action of $S^{+}$can be described similarly, in terms of the fibre functor. Note the stark contrast with the action of the reflection functors of Corollary 2.6, which deals with the case of freely adjoined sinks or sources.

\section{References}

[APR79] M. Auslander, M. I. Platzeck, and I. Reiten, Coxeter functors without diagrams, Trans. Amer. Math. Soc. 250 (1979), 1-46. MR 530043 
[BB76] S. Brenner and M. C. R. Butler, The equivalence of certain functors occurring in the representation theory of Artin algebras and species, J. London Math. Soc. (2) 14 (1976), no. 1, 183-187. MR 0442031

[BBD82] A. A. Bẹ̌linson, J. Bernstein, and P. Deligne, Faisceaux pervers, Analysis and topology on singular spaces, I (Luminy, 1981), Astérisque, vol. 100, Soc. Math. France, Paris, 1982, pp. 5-171. MR 751966

[BGP73] I. N. Bernštĕnn, I. M. Gel'fand, and V. A. Ponomarev, Coxeter functors, and Gabriel's theorem, Uspehi Mat. Nauk 28 (1973), no. 2(170), 19-33. MR 0393065

[BK89] A. I. Bondal and M. M. Kapranov, Representable functors, Serre functors, and reconstructions, Izv. Akad. Nauk SSSR Ser. Mat. 53 (1989), no. 6, 1183-1205, 1337. MR 1039961

[BK90] _ Framed triangulated categories, Mat. Sb. 181 (1990), no. 5, 669-683. MR 1055981

[Gab72] P. Gabriel, Unzerlegbare Darstellungen. I, Manuscripta Math. 6 (1972), 71-103; correction, ibid. 6 (1972), 309. MR 0332887

[GHN17] D. Gepner, R. Haugseng, and T. Nikolaus, Lax colimits and free fibrations in $\infty$-categories, Doc. Math. 22 (2017), 1225-1266. MR 3690268

[GŠs16a] M. Groth and J. Šťovíček, Abstract representation theory of Dynkin quivers of type A, Adv. Math. 293 (2016), 856-941. MR 3474336

[GŠ16b] _ Tilting theory for trees via stable homotopy theory, J. Pure Appl. Algebra 220 (2016), no. 6, 2324-2363. MR 3448799

[GŠ18a] _ Abstract tilting theory for quivers and related categories, Ann. K-Theory 3 (2018), no. 1, 71-124. MR 3695365

[GŠ18b] _ Tilting theory via stable homotopy theory, J. Reine Angew. Math. 743 (2018), 29-90. MR 3859269

[Hap87] D. Happel, On the derived category of a finite-dimensional algebra, Comment. Math. Helv. 62 (1987), no. 3, 339-389. MR 910167

[KL15] A. Kuznetsov and V. A. Lunts, Categorical resolutions of irrational singularities, Int. Math. Res. Not. IMRN (2015), no. 13, 4536-4625. MR 3439086

[Lad07a] S. Ladkani, Universal derived equivalences of posets, arXiv:0705.0946 (2007).

[Lad07b] $\ldots$, Universal derived equivalences of posets of cluster tilting objects, arXiv:0710.2860 (2007).

[Lad07c] _ _ Universal derived equivalences of posets of tilting modules, arXiv:0708.1287 (2007).

[Lad08a] _ Homological properties of finite partially ordered sets, Doctoral thesis, Hebrew University, 2008.

[Lad08b] _ On derived equivalences of categories of sheaves over finite posets, J. Pure Appl. Algebra 212 (2008), no. 2, 435-451. MR 2357344

[Lur09] J. Lurie, Higher topos theory, Annals of Mathematics Studies, vol. 170, Princeton University Press, Princeton, NJ, 2009. MR 2522659

[Lur17] _ Higher algebra, May 2017, Available at the author's homepage.

(Dyckerhoff) Universität Hamburg, Fachbereich Mathematik, Bundesstraße 55, 20146 Hamburg, Germany. E-mail address: tobias.dyckerhoff@uni-hamburg.de

(Jasso) Rheinische Friedrich-Wilhelms-Universität Bonn, Mathematisches Institut, Endenicher Allee 60, 53115 Bonn, Germany. E-mail address: gjasso@math.uni-bonn.de

(Walde) Rheinische Friedrich-Wilhelms-Universität Bonn, Mathematisches Institut, Endenicher Allee 60, 53115 Bonn, Germany. E-mail address: twalde@math.uni-bonn.de 